\title{
Brownian Motion and Negative Curvature
}

\author{
Marc Arnaudon and Anton Thalmaier
}

\begin{abstract}
It is well known that on a Riemannian manifold, there is a deep interplay between geometry, harmonic function theory, and the long-term behaviour of Brownian motion. Negative curvature amplifies the tendency of Brownian motion to exit compact sets and, if topologically possible, to wander out to infinity. On the other hand, non-trivial asymptotic properties of Brownian paths for large time correspond with non-trivial bounded harmonic functions on the manifold. We describe parts of this interplay in the case of negatively curved simply connected Riemannian manifolds. Recent results are related to known properties and old conjectures.
\end{abstract}

Mathematics Subject Classification (2000). Primary 58J65; Secondary 60H30, 31C12, 31C35.

Keywords. Harmonic function, Poisson boundary, Cartan-Hadamard manifold, Conjecture of Greene-Wu, Dirichlet problem at infinity.

\section{Introduction}

In complex analysis the desire to understand how geometry of a complex manifold influences its complex structure has been a guiding inspiration for decades. A typical problem in this direction is the following question.

Question 1 (cf. Wu [33] p.98). If a simply connected complete Kähler manifold has sectional curvature $\leq-c<0$, is it biholomorphic to a bounded domain in $\mathbb{C}^{n}$ ?

Analogous questions may be asked for Riemannian manifolds. The Riemannian counterpart to the above question concerns richness of the space of bounded harmonic functions on simply-connected negatively curved Riemannian manifolds.

Question 2 (cf. Wu [33] p. 139). If $M$ is a simply-connected complete Riemannian manifold with sectional curvature $\leq-c<0$, do there exist $n$ bounded harmonic functions $(n=\operatorname{dim} M)$ which give global coordinates on $M$ ?

Even if complete answers to these questions is not in sight, it seems fair to say that such problems have directly or indirectly inspired a huge part of work 
done in these areas. Concerning Question 2, under the given assumptions, it is still not known in general whether there exist non-trivial bounded harmonic functions at all. This leads us to the famous conjecture of Greene and Wu which claims existence of non-trivial bounded harmonic functions under slightly more refined curvature conditions.

Conjecture (cf. Greene-Wu [13] p. 767). Let $M$ be a simply-connected complete Riemannian manifold of non-positive sectional curvature and $x_{0} \in M$ such that

$$
\operatorname{Sect}_{x}^{M} \leq-\operatorname{cr}(x)^{-2} \quad \text { for all } x \in M \backslash K
$$

for some $K$ compact, $c>0$ and $r=\operatorname{dist}\left(x_{0}, \cdot\right)$. Then $M$ carries non-constant bounded harmonic functions.

From a probabilistic point of view, the conjecture of Greene and $\mathrm{Wu}$ concerns the eventual behaviour of Brownian motion on Cartan-Hadamard manifolds as time tends to infinity. We briefly sketch the relation with Brownian motion. Indeed, for any Riemannian manifold, we have the following well-known probabilistic characterization.

Lemma 1.1. For a Riemannian manifold $(M, g)$ the following two conditions are equivalent:

i) There exist non-constant bounded harmonic functions on $M$.

ii) Brownian on $(M, g)$ has non-trivial exit sets, i.e., if $X$ is a Brownian motion on $M$ then there exist open subsets $U$ in the 1-point compactification $\hat{M}$ of $M$ such that

$$
\mathbb{P}\left\{X_{t} \in U \text { eventually }\right\} \neq 0 \text { or } 1 .
$$

More precisely, Brownian motion $X$ on $M$ may be realized on the space $C\left(\mathbb{R}_{+}, \hat{M}\right)$ of continuous paths with values in the 1-point compactification $\hat{M}$ of $M$, equipped with the filtration $\mathscr{F}_{t}=\sigma\left\{X_{s}=\operatorname{pr}_{s} \mid s \leq t\right\}$ generated by the coordinate projections $\operatorname{pr}_{s}$ up to time $t$. Let

$$
\zeta=\sup \left\{t>0: X_{t} \in M\right\}
$$

be the lifetime of $X$ and let $\mathscr{F}_{\text {inv }}$ denote the shift-invariant $\sigma$-field on $C\left(\mathbb{R}_{+}, \hat{M}\right)$. Then there is a canonical isomorphism between the space $\mathscr{H}_{\mathrm{b}}(M)$ of bounded harmonic functions on $M$ and the set $b \mathscr{F}_{\text {inv }}$ of bounded $\mathscr{F}_{\text {inv-measurable random }}$ variables up to equivalence, given as follows:

$$
\mathscr{H}_{\mathrm{b}}(M) \stackrel{\sim}{\longrightarrow} b \mathscr{F}_{\text {inv }} / \sim, \quad u \longmapsto \lim _{t \uparrow \zeta}\left(u \circ X_{t}\right) .
$$

(Bounded shift-invariant random variables are considered as equivalent, if they agree $\mathbb{P}_{x}$-a.e., for each $x \in M$. Here $\mathbb{P}_{x}$ denotes the law of Brownian motion starting at $x$.)

Note that the isomorphism (1.1) is well defined by the martingale convergence theorem; the inverse map to (1.1) is given by taking expectations:

$$
b \mathscr{F}_{\text {inv }} / \sim \ni H \longmapsto u \in \mathscr{H}_{\mathrm{b}}(M) \text { where } u(x):=\mathbb{E}_{x}[H] .
$$


In particular,

$$
u(x):=\mathbb{P}_{x}\left\{X_{t} \in U \text { eventually }\right\}
$$

defines a bounded harmonic function on $M$, which is non-constant if and only if $U$ is a non-trivial exit set. Clearly $\left\{X_{t} \in U\right.$ eventually $\} \in \mathscr{F}$ inv .

Conversely, to an element $B \in \mathscr{F}_{\text {inv }}$ one can associate an exit set $U$ such that $\left\{X_{t} \in U\right.$ eventually $\}=B$. For instance, one may take $U=\{x \in M: u(x)>1 / 2\}$, where $u$ is the harmonic map defined by $u(x)=\mathbb{P}_{x}(B)$.

\section{Brownian motion on rotationally symmetric manifolds}

In this section we determine the asymptotic behaviour of Brownian motion on rotationally symmetric Riemannian manifolds, see [26, 14]. Such manifolds play an important role as comparison models for more general manifolds.

\subsection{Rotationally symmetric manifolds}

Let $(M, g)$ be a Riemannian manifold of dimension $n \geq 2$ and $x \in M$ such that the exponential map $\exp _{x}$ defines a diffeomorphism between $T_{x} M$ and $M$. The manifold $M$ is then diffeomorphic to $\mathbb{R}^{n}$; in geodesic polar coordinates on $M \backslash\{0\}=$ ] $0, \infty\left[\times S^{n-1}\right.$ about $0 \in M$ the metric takes the form

$$
g=d r \otimes d r+h_{r}
$$

with Riemannian metrics $h_{r}$ on $S^{n-1}$ depending on the radius $r$.

We consider the following two particular cases:

(a) $g=d r \otimes d r+f^{2}(r, \cdot) h$ where $\left.f:\right] 0, \infty\left[\times S^{n-1} \rightarrow\right] 0, \infty[$ is a scalar function and $h$ a fixed Riemannian metric on $S^{n-1}$ (independent of $r$ );

(b) $g=d r \otimes d r+f^{2}(r) d \vartheta^{2}$ where $d \vartheta^{2}$ is the standard metric on $S^{n-1}$ and $f:] 0, \infty[\rightarrow] 0, \infty[$.

Case (b) corresponds to the case of rotationally invariant models, see [13]. Let

$$
q: M \backslash\{0\} \rightarrow S^{n-1}, \quad(r, \vartheta) \mapsto \vartheta .
$$

The following Lemma is immediately verified.

Lemma 2.1. Let $q: M \backslash\{0\} \rightarrow S^{n-1}$ be the angular map (2.1).

(i) In situation (a) the map $q:(M \backslash\{0\}, g) \rightarrow\left(S^{n-1}, h\right)$ is harmonic if and only if

$$
(n-3) \operatorname{grad} f_{r}=0
$$

where grad $f_{r}$ denotes the gradient vector field to $f_{r}=f(r, \cdot)$ on $\left(S^{n-1}, h\right)$.

(ii) In situation (b) the map $q:(M \backslash\{0\}, g) \rightarrow\left(S^{n-1}, d \vartheta^{2}\right)$ is affine, and moreover a harmonic morphism with dilatation $f^{-1}$, i.e.,

$$
\Delta_{M}(\varphi \circ q)=f^{-2}\left(\Delta_{S^{n-1}} \varphi\right) \circ q, \quad \varphi \in C^{\infty}\left(S^{n-1}\right),
$$

where $\Delta_{M}$ and $\Delta_{S^{n-1}}$ are the Laplacians on $(M, g)$, resp., $\left(S^{n-1}, d \vartheta^{2}\right)$.

We can give a complete description of the behaviour of Brownian motion on rotationally invariant manifolds, see [14] for details. 
Theorem 2.2 (Brownian motion on rotationally invariant models). Let $M$ be a rotationally invariant model with center $0 \in M$ and metric

$$
g=d r \otimes d r+f^{2}(r) d \vartheta^{2} .
$$

Let $X$ be Brownian motion on $(M, g)$ with $X_{0}=x_{0}\left(x_{0} \neq 0\right)$ which is decomposed according to $M \backslash\{0\}=] 0, \infty\left[\times S^{n-1}\right.$ into its radial and angular process

$$
X=(r(X), \vartheta(X)) \text {. }
$$

(i) For the radial process, we have $r(X)_{t} \rightarrow \infty$ a.s. (i.e., $X$ is transient) if and only if

$$
\int_{1}^{\infty} f^{1-n}(r) d r<\infty
$$

(ii) The lifetime $\zeta$ of $X$ is either a.s. finite or a.s. infinite, and a.s. finite if and only if

$$
\int_{1}^{\infty} f^{n-1}(r)\left\{\int_{r}^{\infty} f^{1-n}(\rho) d \rho\right\} d r<\infty .
$$

(iii) The angular process $\vartheta(X)$ converges on $S^{n-1}$ for $t \nearrow \zeta$ a.s., if and only if

$$
\int_{1}^{\infty} f^{n-3}(r)\left\{\int_{r}^{\infty} f^{1-n}(\rho) d \rho\right\} d r<\infty .
$$

The latter is equivalent to $M$ being a non Liouville manifold.

Sketch of Proof. (1) Denote by $R_{t}:=r(X)_{t}$ and $\Theta_{t}:=\vartheta(X)_{t}$ the radial part, respectively angular part of $X$. Then

$$
d R=d W+\frac{1}{2}(\Delta r \circ X) d t
$$

for some one-dimensional Brownian motion $W$. Since $(\Delta r)(X)=(n-1)\left(f^{\prime} / f\right)(R)$, the radial process $R$ solves the SDE

$$
d R=d W+\frac{1}{2}\left[(n-1)\left(f^{\prime} / f\right) \circ R\right] d t .
$$

Hence $R$ is a one-dimensional diffusion on $] 0, \infty[$ with infinitesimal generator

$$
\frac{1}{2}\left\{D^{2}+(n-1)\left(f^{\prime} / f\right) D\right\}, \quad D:=d / d t .
$$

We may calculate the Riemannian quadratic variation of $X$ (see for instance [12]) using the relation

$$
d[X, X]=d[R, R]+f^{2}(R) d[\Theta, \Theta] .
$$

Taking into account that $d[X, X]=(\operatorname{dim} M) d t=n d t$ and $d[R, R]=d t$, we arrive at

$$
d[\Theta, \Theta]=(n-1)\left(f^{-2} \circ R\right) d t
$$


According to the Martingale Convergence Theorem of Darling-Zheng (see [12]) the process $\Theta$ converges as $t \nearrow \zeta$ on $S^{n-1}$ a.s. if and only if $\int_{0}^{\zeta} f^{-2}\left(R_{t}\right) d t<\infty$ a.s. Let

$$
T(t)=\int_{0}^{t} f^{-2}\left(R_{s}\right) d s, \quad t<\zeta,
$$

and consider the continuous time change

$$
\tau_{t}:=T^{-1}(t) \equiv \inf \left\{s \in \mathbb{R}_{+}: T(s) \geq t\right\}, \quad t<T_{\zeta}:=T(\zeta) .
$$

Obviously $T_{\zeta}$ is the maximal lifetime of the time-changed radial process $\tilde{R}_{t}:=R_{\tau_{t}}$, with the consequence that $\Theta$ converges as $t \nearrow \zeta$ if and only if the lifetime of $\tilde{R}$ is finite a.s.

(2) Note that by Lemma 2.1, the angular map $q$ is a harmonic morphism with dilatation $f^{-1}$. Hence $X$ decomposes as

$$
X_{t}=\left(R_{t}, B_{T(t)}\right)
$$

for some Brownian motion $B$ on $\left(S^{n-1}, d \vartheta^{2}\right)$. In other words, by means of the time change $\left(\tau_{t}\right)$, radial and angular part of $X$ decouple as

$$
X_{\tau_{t}}=\left(R_{\tau_{t}}, B_{t}\right), \quad t<T_{\zeta} .
$$

In the new time scale the angular component is just Brownian motion on $\left(S^{d-1}, d \vartheta^{2}\right)$ which runs up to time $T_{\zeta}$. In particular, the angular part converges if and only if $T_{\zeta}$ is finite a.s.

(3) Since $R$ is a one-dimensional diffusion on ]0, $\infty$ [ with generator

$$
\frac{1}{2}\left\{D^{2}+(d-1)\left(f^{\prime} / f\right) D\right\}
$$

the time-changed radial process $\tilde{R}$ is generated by

$$
\frac{1}{2} f^{2}\left\{D^{2}+(d-1)\left(f^{\prime} / f\right) D\right\} .
$$

All questions of interest concerning transience and lifetime of $R$, resp. $\tilde{R}$, can now be answered by means of the classical Feller theory of one-dimensional diffusions.

Recall that a Riemannian manifold $M$ is called Liouville manifold, if all bounded harmonic functions on $M$ are constant. Note that in dimension 2, by Theorem 2.2, a rotationally invariant model $M$ is Liouville if and only if Brownian motion on $M$ is recurrent.

The radial curvature of $M$ at a point $x$ is by definition the restriction of the sectional curvature to radial planes $E$, i.e., planes $E \subset T_{x} M$ containing the radial vector $(\partial / \partial r)_{x}$. For rotationally invariant models $M$ the radial curvature at any point $x$ obviously depends only on the radius $r=r(x)$. We denote

$$
k_{M}(r):=\text { radial curvature at } x \in M \text { if } r(x)=r \text {. }
$$


For a rotationally invariant model $M$ with metric $g=d r \otimes d r+f^{2}(r) d \vartheta^{2}$, one verifies immediately

$$
k_{M}=-f^{\prime \prime} / f .
$$

\subsection{Curvature conditions for the Liouville property}

The conditions of Theorem 2.2 are easily rewritten in terms of radial curvature, see [14] for instance. It is interesting to note that no lower curvature bounds are required for the existence of non-trivial bounded harmonic functions.

Theorem 2.3 (Bounded harmonic functions on rotationally symmetric manifolds). Let $(M, g)$ be a rotationally symmetric model with center $0 \in M$ and Riemannian metric

$$
g=d r \otimes d r+f^{2}(r) d \vartheta^{2} .
$$

Let

$$
k_{M}(r)=-f^{\prime \prime}(r) / f(r)
$$

be the radial curvature function of $(M, g)$. Suppose that $k_{M}(\cdot) \leq 0$. Further let $c=1$ in case of $n=2$, resp., $c=1 / 2$ in case of $n \geq 3$.

A. If for some $\varepsilon>0$,

$$
k_{M}(r) \leq-\frac{(c+\varepsilon)}{r^{2} \log r}, \quad \text { for } r \text { sufficiently large, }
$$

then $M$ carries non-constant bounded harmonic functions.

B. If however for some $\varepsilon>0$,

$$
k_{M}(r) \geq-\frac{(c-\varepsilon)}{r^{2} \log r}, \quad \text { for } r \text { sufficiently large, }
$$

then $M$ is a Liouville manifold.

Remark 2.4. Note that constant negative curvature outside a compact set is not sufficient for existence of non-constant bounded harmonic functions, not even for hyperbolicity (i.e., transience of Brownian motion), as the following example shows. Let $M=\mathbb{R}^{2}$ be equipped with a rotationally symmetric metric, for instance, given by the radial function

$$
f(r)=\exp (-r)
$$

for $r>1$ and a differentiable interpolation for $0 \leq r \leq 1$, such that $f(0)=0$ and $f^{\prime}(0)=1$ holds. Then $(M, g)$ has constant negative curvature outside the unit disk, but according to Theorem 2.2, Brownian motion on $(M, g)$ is recurrent; the manifold $M$ is hence Liouville. 


\section{Brownian motion on Cartan-Hadamard manifolds of pinched negative curvature}

Let $(M, g)$ be a Cartan-Hadamard manifold, i.e., a simply connected, metrically complete Riemannian manifold of non-positive sectional curvature. (All manifolds in the sequel are supposed to be connected.) In terms of the exponential map $\exp _{x_{0}}: T_{x_{0}} M \stackrel{\sim}{\longrightarrow} M$ at a fixed base point $x_{0} \in M$, we identify

$$
\rho: \mathbb{R}^{n} \cong T_{x_{0}} M \stackrel{\sim}{\longrightarrow} M .
$$

Via pullback of the metric $g$ on $M$ to $\mathbb{R}^{n}$, we obtain an isometric isomorphism $(M, g) \cong\left(\mathbb{R}^{n}, \rho^{*} g\right)$; in particular, $\left.M \backslash\left\{x_{0}\right\} \cong\right] 0, \infty\left[\times S^{n-1}\right.$. In terms of such global polar coordinates, Brownian motion $X$ on $M$ will be decomposed into its radial and angular part,

$$
X_{t}=\left(r\left(X_{t}\right), \vartheta\left(X_{t}\right)\right)
$$

where $r\left(X_{t}\right)=\operatorname{dist}\left(x_{0}, X_{t}\right)$ and where $\vartheta\left(X_{t}\right)$ takes values in $S^{n-1}$.

\subsection{Boundaries at infinity}

For a Cartan-Hadamard manifold $M$ of dimension $n$ there is a natural geometric compactification which is given by attaching an ideal boundary $M(\infty)$ at infinity. Formally the points of the boundary are given as equivalence classes of geodesics which stay in bounded distance,

$$
\bar{M}=M \dot{\cup} M(\infty)
$$

where $M(\infty)=\{\gamma: \mathbb{R} \rightarrow M \mid \gamma$ geodesics $\} / \sim$ and

$$
\gamma_{1} \sim \gamma_{2} \Leftrightarrow \limsup _{t \rightarrow \infty} \operatorname{dist}\left(\gamma_{1}(t), \gamma_{2}(t)\right)<\infty .
$$

Then $M \cup M(\infty)$ equipped with the cone topology is homeomorphic to the closed unit ball $B \subset \mathbb{R}^{n}$ with boundary $\partial B=S^{n-1}$, cf. [6, 11], whereas $M$ itself is diffeomorphic to the open unit ball. In particular,

$$
S_{\infty}(M):=M(\infty) \equiv\{\gamma(\infty) \mid \gamma \text { geodesics }\}
$$

is a $(n-1)$-sphere which serves as horizon at infinity. In terms of polar coordinates on $M$, a sequence $\left(r_{n}, \vartheta_{n}\right)_{n \in \mathbb{N}}$ of points in $M$ converges to a point on the sphere at infinity $S_{\infty}(M)$ if and only if $r_{n} \rightarrow \infty$ and $\vartheta_{n} \rightarrow \vartheta \in S^{n-1}$.

Definition 3.1. Let $M$ be a Cartan-Hadamard manifold and $f: S_{\infty}(M) \rightarrow \mathbb{R}$ be a continuous function defined on the sphere at infinity. The Dirichlet problem at infinity is to find a harmonic function $h: M \rightarrow \mathbb{R}$ which extends continuously to $S_{\infty}(M)$ and coincides there with the given function $f$, i.e.,

$$
h \mid S_{\infty}(M)=f .
$$

The Dirichlet problem at infinity is said to be solvable if this is possible for every such function $f$. 
Solvability of the Dirichlet problem at infinity provides a rich class of nontrivial bounded harmonic functions on $M$, given as solutions of the Dirichlet problem at infinity for various boundary functions.

\subsection{Angular convergence and solvability of the Dirichlet problem at infinity}

First results concerning angular convergence have been obtained by J.-J. Prat $[29,30]$. He proved that on a Cartan-Hadamard manifold with sectional curvature bounded from above by a negative constant $-b^{2}, b>0$, Brownian motion is transient, i.e., almost surely all paths of the Brownian motion exit $M$ at the sphere at infinity [30]. If in addition the sectional curvatures are bounded from below by a constant $-a^{2}, a>b$, he showed that the angular part $\vartheta\left(X_{t}\right)$ of the Brownian motion almost surely converges as $t \rightarrow \zeta$.

Y. Kifer [21] presented a stochastic proof that on Cartan-Hadamard manifolds with sectional curvature pinched between two strictly negative constants and satisfying a certain additional technical condition, the Dirichlet problem at infinity has a unique solution. The proof there was given in explicit terms for the two-dimensional case.

D. Sullivan [31] finally gave a complete stochastic proof of the fact that on a Cartan-Hadamard manifold with pinched negative curvature the Dirichlet problem at infinity is uniquely solvable. The crucial open point has been to show that the harmonic measure class is non-trivial in this case.

Theorem 3.2 (J.-J. Prat [30], Y. Kifer [21]). Let $(M, g)$ be a Cartan-Hadamard manifold of dimension $n$ such that

$$
-a^{2} \leq \operatorname{Sect}^{M} \leq-b^{2}<0 .
$$

Let $X_{t}=\left(r\left(X_{t}\right), \vartheta\left(X_{t}\right)\right)$ be a Brownian motion on $M$ decomposed in its radial and angular part. Then a.s., as $t \rightarrow \infty$,

$$
r\left(X_{t}\right) \rightarrow \infty, \text { and } \vartheta\left(X_{t}\right) \text { converges on } S^{n-1} .
$$

Denoting $\Theta_{\infty}:=\lim _{t \rightarrow \infty} \vartheta\left(X_{t}\right)$ and $\mu_{x}:=\mathbb{P}_{x} \circ \Theta_{\infty}^{-1}$, for every Borel set $U \subset$ $S_{\infty}(M)$, the function

$$
u(x)=\mathbb{P}_{x}\left\{\Theta_{\infty} \in U\right\} \equiv \mu_{x}(U) \text { is harmonic on } M .
$$

Remark 3.3. By the maximum principle, the Poisson hitting measure at infinity are equivalent,

$$
\mu_{x} \sim \mu_{y} \quad \text { for } x, y \in M,
$$

and define a measure class on the sphere at infinity. Indeed, for any Borel set $U \subset S_{\infty}(M)$, the assignment

$$
h_{U}: x \mapsto \mu_{x}(U)
$$

defines a bounded harmonic function on $M$, which by the maximum principle is either identically equal to 0 or 1 or takes its values in $] 0,1[$. For the solvability of the Dirichlet problem at infinity this measure class $\mu$ must be shown to be non-trivial. 
Theorem 3.4 (D. Sullivan [31], M. T. Anderson [3]). Let (M,g) be a CartanHadamard manifold of dimension $n$ such that

$$
-a^{2} \leq \operatorname{Sect}^{M} \leq-b^{2}<0 .
$$

The harmonic measure class on $S_{\infty}(M)$ is positive on each non-void open set. In fact, if $x_{i}$ in $M$ converges to $x_{\infty}$ in $S_{\infty}(M)$, then the Poisson hitting measures $\mu_{x_{i}}$ tend weakly to the Dirac mass at $x_{\infty}$.

Corollary 3.5 (Solvability of the Dirichlet problem at infinity). Let $(M, g)$ be a $n$ dimensional Cartan-Hadamard manifold with sectional curvature pinched between to negative constants,

$$
-a^{2} \leq \operatorname{Sect}^{M} \leq-b^{2}<0
$$

For $f \in C\left(S_{\infty}(M)\right)$ let

$$
u(x)=\mathbb{E}_{x}\left[f\left(\Theta_{\infty}\right)\right] .
$$

Then

$$
u \in C(\bar{M}), \quad \Delta u=0 \text { on } M \quad \text { and } \quad u \mid S_{\infty}(M)=f .
$$

Denoting by $\mathscr{H}_{b}(M)$ the Banach space of bounded harmonic functions on $M$, a complete description of bounded harmonic functions on $M$ has been given by M.T. Anderson [3].

It is an interesting feature of the pinched curvature case that any bounded harmonic function on $M$ comes from a solution of the Dirichlet problem at $\infty$ (for some bounded measurable $f$ ).

Theorem 3.6 (M.T. Anderson [3]). Let $(M, g)$ be a Cartan-Hadamard manifold of dimension $n \geq 2$, whose sectional curvatures satisfy $-a^{2} \leq \operatorname{Sect}_{x}^{M} \leq-b^{2}<0$ for all $x \in M$. Then the linear mapping

$$
\begin{aligned}
P: L^{\infty}\left(S_{\infty}(M), \mu\right) & \rightarrow \mathscr{H}_{b}(M), \\
f & \mapsto P(f), \quad P(f)(x):=\int_{S_{\infty}(M)} f d \mu_{x}
\end{aligned}
$$

is a norm-nonincreasing isomorphism onto $\mathscr{H}_{b}(M)$.

In the case of a Cartan-Hadamard manifold of pinched negative curvature the limiting angle $\Theta_{\infty}=\lim _{t \rightarrow \infty} \vartheta\left(X_{t}\right)$ generates the shift-invariant $\sigma$-field of $X$,

$$
\mathscr{F}_{\text {inv }}=\sigma\left(\Theta_{\infty}\right) \text {. }
$$

Thus all shift-invariant properties which allow to distinguish Brownian paths for large times, are expressible in terms of the angular projection of $X$ onto $S_{\infty}(M)$.

Remark 3.7 (Probabilistic conditions for the solvability of the Dirichlet problem at $\infty)$. The proof of solvability of the Dirichlet problem at $\infty$ by probabilistic methods proceeds in several steps:

1. Prove transience of Brownian motion, i.e., $r\left(X_{t}\right) \rightarrow \infty$ for the radial part of Brownian motion. 
2. Establish convergence of the angular part $\vartheta\left(X_{t}\right)$ of Brownian motion; this provides entrance measures $\mu_{x}$ into the sphere at $\infty$.

3. Show that the harmonic measure class at infinity is non-trivial and all boundary points are regular, i.e.,

$$
\mu_{x_{n}} \rightarrow \delta_{x_{\infty}} \text { in probability, as } x_{n} \rightarrow x_{\infty} \in S_{\infty}(M)
$$

The upper curvature bound in this approach guarantees a certain escape rate for Brownian motion, whereas the lower curvature bound controls the angular oscillations.

The constant curvature bounds for solvability of the Dirichlet problem have been relaxed in various directions, e.g., Hsu-March [18], Hsu [16], Vähäkangas [32], Holopainen-Vähäkangas [15].

It is interesting to note that the curvature may well tend to $-\infty$ as long the oscillation of curvature (in the sense of the quotient of lower and upper bounds) is bounded.

Theorem 3.8 (Vähäkangas [32], Holopainen-Vähäkangas [15]). Let $M$ be a simplyconnected complete Riemannian manifold of non-positive sectional curvature such that

$$
\operatorname{Sect}_{x}^{M} \leq-\frac{c}{r^{2}(x)} \quad \forall x \in M \backslash K
$$

for some $K$ compact in $M, c>0$ and $r=\operatorname{dist}\left(x_{0}, \cdot\right)$. Suppose that there exists $C>1$ such that for each $x \in M \backslash K$ and all radial planes $E, E^{\prime} \subset T_{x} M$

$$
\left|\operatorname{Sect}_{x}^{M}(E)\right| \leq C\left|\operatorname{Sect}_{x}^{M}\left(E^{\prime}\right)\right| .
$$

Then the Dirichlet problem at $\infty$ is solvable.

\subsection{The role of lower curvature bounds}

The discussion above raises the question to what extent lower curvature bounds are necessary.

In the special case of a Riemannian surface $M$ of negative curvature bounded from above by a negative constant, Kendall [20] gave a simple stochastic argument that the Dirichlet problem at infinity is uniquely solvable. He used the fact that every geodesic on the Riemannian surface connecting two different points on the sphere at infinity divides the surface into two totally geodesic disjoint half-parts. Starting from a point $x$ on $M$, with non-trivial probability, Brownian motion will eventually stay in one of the two half-parts up to its lifetime. As this is valid for every geodesic and every starting point $x$, the non-triviality of the harmonic measure class on $S_{\infty}(M)$ easily follows.

Choi [8] provided a general criterion, the so-called convex conic neighbourhood condition, which is sufficient for solvability of the Dirichlet problem at infinity. The condition requires that distinct points $x, y \in S_{\infty}(M)$ can be separated in $\bar{M}$ by convex sets. 
Definition 3.9. A Cartan-Hadamard manifold $M$ satisfies the convex conic neighbourhood condition at $x \in S_{\infty}(M)$ if for any $y \in S_{\infty}(M), y \neq x$, there exist subsets $V_{x}$ and $V_{y}$ of $\bar{M}$, containing $x$ and $y$ respectively, such that $V_{x}$ and $V_{y}$ are disjoint open sets of $\bar{M}$ in terms of the cone topology and $V_{x} \cap M$ is convex with $C^{2}$-boundary. If this condition holds for all $x \in S_{\infty}(M), M$ is said to satisfy the convex conic neighbourhood condition.

Theorem 3.10 (H. I. Choi [8]). Let $M$ be a Cartan-Hadamard manifold satisfying the convex conic neighbourhood condition, with Sect $^{M} \leq c$ for some constant $c<0$. Then the Dirichlet problem at infinity for $M$ is solvable.

Intuitively the convex conic neighbourhood condition looks as a plausible assumption suggesting that lower curvature bounds might be dispensable. It has been shown by Ancona [2] that this is however not the case. In [2] he constructed an example of a complete, simply connected Riemannian manifold $M$ of dimension 3 , with $\operatorname{Sect}^{M} \leq-1$, and a point $\infty_{M} \in S_{\infty}(M)$ such that

(i) Brownian motion on $M$ has a.s. infinite lifetime;

(ii) With probability 1 , any Brownian motion on exits from $M$ at $\infty_{M}$.

In addition, the convex hull of any neighbourhood of $\infty_{M}$ in $\bar{M}$ contains all of $M$.

Remark 3.11. It is obvious that on Ancona's manifold the Dirichlet problem at infinity is not solvable. Indeed, let

$$
f \in C\left(S_{\infty}(M)\right), u \in C(\bar{M}) \text { such that } \Delta u=0 \text { on } M \text { and } u \mid S_{\infty}(M)=f .
$$

Then, for any $x \in M$,

$$
u(x)=\mathbb{E}_{x}\left[f\left(X_{\infty}\right)\right]=f\left(\infty_{M}\right)
$$

which shows that the function $u$ must be constant. Hence only the constant harmonic functions have a continuous continuation to the boundary at $\infty$.

Nevertheless the above discussion does not answer the question whether there exist non-trivial bounded harmonic functions on Ancona's manifold, since there may be non-trivial bounded harmonic functions which do not come from boundary values at infinity. Ancona did not address this problem in his paper [2]. It turns out that there are indeed plenty of non-trivial bounded harmonic functions with no continuation to the boundary at infinity, as will be discussed in the next section.

Borbély [7] constructed a similar example of a Cartan-Hadamard manifold with curvature bounded above by a strictly negative constant, on which the Dirichlet problem at infinity is not solvable. Borbély does not discuss the behaviour of Brownian motion on his manifold. However it turns out that from a probabilistic point of view, the manifolds of Ancona and Borbély share similar properties. Borbély's construction is however technically simpler; in the next section we shall give a detailed probabilistic analysis of his manifold. 


\section{Brownian motion on Cartan-Hadamard manifolds of unbounded negative curvature}

In this section we discuss examples of negatively curved manifolds where the potential theoretic boundary does not coincide with the geometric boundary at infinity. To see the full Poisson boundary, certain points at infinity need to be blown up in a non-trivial way. Such examples indicate that the situation concerning the conjecture of Greene and Wu about existence of non-trivial bounded harmonic functions on Cartan-Hadamard manifolds may be more complicated than expected.

\subsection{Description of the manifold}

The construction of the following example is basically due to Borbély [7]. Let

$$
\mathbb{H}^{2}=\left\{(x, y) \in \mathbb{R}^{2} \mid y>0\right\}
$$

be the hyperbolic half-plane equipped with the hyperbolic metric $d s_{\mathbb{H}^{2}}^{2}$ of constant curvature -1 . Denote by $L=\{(0, y) \mid y>0\}$ the positive $y$-axis in $\mathbb{H}^{2}$ and let $H$ denote one component of $\mathbb{H}^{2} \backslash L$. We shall define a Riemannian manifold $M$ as the warped product:

$$
M:=(H \cup L) \times{ }_{g} S^{1},
$$

with Riemannian metric

$$
d s_{M}^{2}=d s_{\mathbb{H}^{2}}^{2}+g d s_{S^{1}}^{2},
$$

where $g: H \cup L \rightarrow \mathbb{R}_{+}$is a positive $C^{\infty}$-function which has to be chosen appropriately. By identifying points $\left(\ell, \alpha_{1}\right)$ and $\left(\ell, \alpha_{2}\right)$ with $\ell \in L$ and $\alpha_{1}, \alpha_{2} \in S^{1}$, we make $M$ a simply connected space.

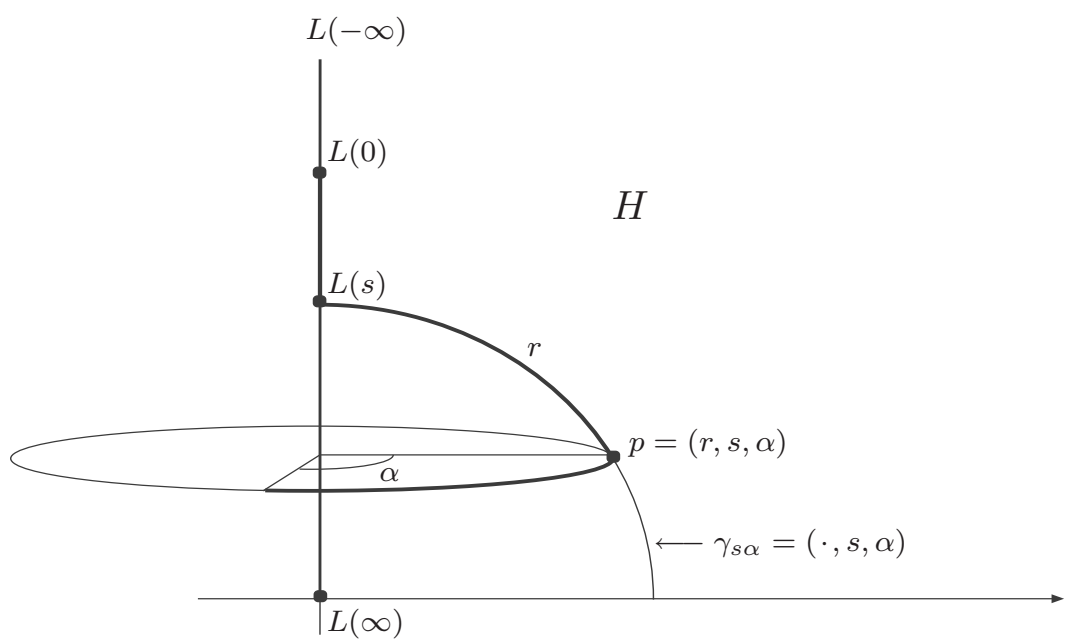

Figure 1. Fermi coordinates $(r, s, \alpha)$ for $M$ 
We introduce Fermi coordinates $(r, s, \alpha)$ on $M$, where for a point $p \in M$ the coordinate $r$ is the hyperbolic distance between $p$ and the geodesic $L$, i.e., the hyperbolic length of the perpendicular on $L$ through $p$. The coordinate $s$ is the parameter on the geodesic $\{L(s): s \in]-\infty, \infty[\}$, i.e., the length of the geodesic segment on $L$ joining $L(0)$ and the orthogonal projection $L(s)$ of $p$ onto $L$. Furthermore, $\alpha \in\left[0,2 \pi\right.$ [ is the parameter on $S^{1}$ when using the parameterization $e^{i \alpha}$ of $S^{1}$.

Theorem 4.1. Let $M=(H \cup L) \times{ }_{g} S^{1}$ be equipped with the metric

$$
d s_{M}^{2}=d s_{\mathbb{H}^{2}}^{2}+g d s_{S^{1}}^{2} .
$$

There exists a choice for a smooth wedge function $g: \mathbb{R}_{+} \times \mathbb{R} \rightarrow \mathbb{R}_{+}$such that the following two properties hold true:

(i) $\left(M, d s_{M}^{2}\right)$ is Cartan-Hadamard with Sect $^{M} \leq-1$;

(ii) $X_{t} \rightarrow L(+\infty)$ a.s. for each Brownian motion $X$ on $M$.

Remark 4.2. Typical geodesics in $M$ are the ones of the type $\gamma_{s \alpha}: r \mapsto(r, s, \alpha)$. We get the sphere at infinity $S_{\infty}(M)$ by following these geodesics to infinity, together with the endpoints $L(-\infty), L(+\infty)$ of the vertical geodesic $L$ which serves as symmetry axis:

$$
S_{\infty}(M)=\left\{\gamma_{s \alpha}(\infty) \mid s \in \mathbb{R}, \alpha \in[0,2 \pi[\} \cup\{L( \pm \infty)\}\right.
$$

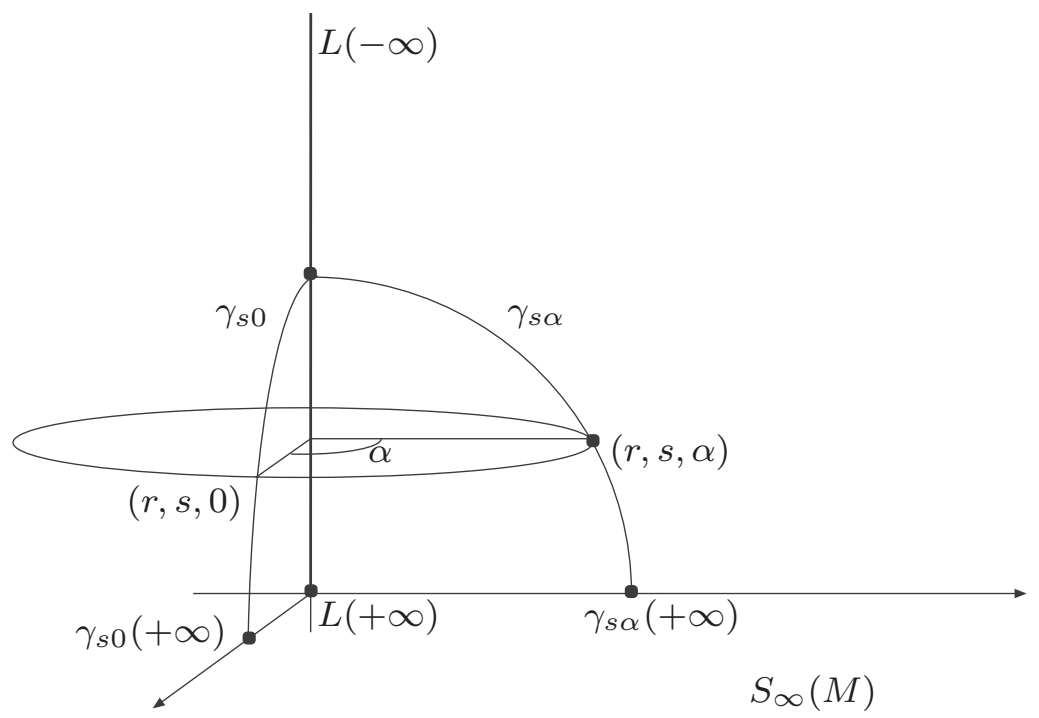

Figure 2. Sphere at infinity $S_{\infty}(M)$ 


\subsection{Asymptotic behaviour of Brownian motion}

The behaviour of Brownian motion on $M$ may be investigated in the given coordinates, by studying the corresponding stochastic differential equations. It turns out that Brownian motion almost surely exits the manifold at a distinguished point of the sphere at infinity, independently of its starting point $x$ at time 0 .

Theorem 4.3 (Arnaudon-Thalmaier-Ulsamer [5]). Writing Brownian motion on $M$ in terms of the Fermi coordinates $X_{t}=\left(R_{t}, S_{t}, A_{t}\right)$, we have the following asymptotic properties, as $t$ approaches the lifetime $\zeta$ of $X$,

$$
\text { (i) } R_{t} \rightarrow+\infty \text { a.s. (ii) } S_{t} \rightarrow+\infty \text { a.s. (iii) } A_{t} \rightarrow A_{\zeta} \text { a.s., }
$$

where the limiting variable $A_{\zeta}$ is a non-trivial random variable taking values in $S^{1}$. Moreover, for any $x \in M$, the support of $\mathbb{P}_{x} \circ\left(A_{\zeta}\right)^{-1}$ equals all of $S^{1}$.

Remark 4.4. An immediate consequence of Theorem 4.3 is that, for each nonconstant $f \in C\left(S^{1} ; \mathbb{R}\right)$, the formula

$$
h(x)=\mathbb{E}_{x}\left[f\left(A_{\zeta}\right)\right]
$$

defines a non-trivial bounded harmonic function $h$ on $M$. The variable $A_{\zeta}$ gives the asymptotic direction on the sphere at $\infty$ along which Brownian motion approaches the point

$$
\infty_{M}=L(+\infty) \in S_{\infty}(M) .
$$

The question whether $M$ carries further non-trivial bounded harmonic functions besides functions of the type (4.1), turns out to be highly non-trivial. To answer this question we go back to equations of Brownian motion in the specified Fermi coordinates

$$
\left\{\begin{aligned}
d R & =F^{1}(R, S) d t+d M^{1} \\
d S & =F^{2}(R, S) d t+d M^{2} \\
d A & =\quad d M^{3}
\end{aligned}\right.
$$

with $M^{1}, M^{2}$ and $M^{3}$ local martingales. After an appropriate change of time (which turns the drift of $R$ into a deterministic linear motion) this system writes as

$$
\left\{\begin{array}{l}
d \hat{R}=d t+d \hat{M}^{1} \\
d \hat{S}=f(\hat{R}, \hat{S}) d t+d \hat{M}^{2} \\
d \hat{A}=\quad d \hat{M}^{3}
\end{array}\right.
$$

where the local martingale parts $\hat{M}_{t}^{i}$ converge as $t \rightarrow \zeta$.

The idea now is to compare the asymptotic behaviour of $(R, S)$ with the deterministic dynamical system in the $(r, s)$-plan given by

$$
\left\{\begin{array} { l } 
{ d r _ { t } = d t } \\
{ d s _ { t } = f ( r _ { t } , s _ { t } ) d t }
\end{array} \quad \text { i.e., } \quad \left\{\begin{array}{l}
\dot{r}_{t}=1 \\
\dot{s}_{t}=f\left(t, s_{t}\right) .
\end{array}\right.\right.
$$


It seems reasonable to expect that

$$
\left(\hat{R}_{t}, \hat{S}_{t},\right) \approx\left(r_{t}, s_{t}\right), \quad \text { for } t \gg 0,
$$

where $\left(r_{t}, s_{t}\right)$ is an integral curve of the vector field

$$
V=\frac{\partial}{\partial r}+f(r, s) \frac{\partial}{\partial s}
$$

Taking into account the fact that there is a good approximation $q=q(r)$ such that

$$
f(r, s) \approx q(r) \text { for large } r
$$

we consider

$$
\Gamma_{s_{0}}(t)=\left(t, s_{0}+\int_{0}^{t} q(u) d u\right)
$$

and let

$$
\Gamma_{s_{0}}:=\left\{\Gamma_{s_{0}}(t): t \geq 0\right\} .
$$

Then $\left(\Gamma_{s_{0}}\right)_{s_{0} \in \mathbb{R}}$ defines a foliation of $H$. The change of coordinates:

$$
\begin{aligned}
\Phi: & \mathbb{R}_{+} \times \mathbb{R} \rightarrow \mathbb{R}_{+} \times \mathbb{R}, \\
& (r, s) \rightarrow\left(r, s-\int_{0}^{r} q(u) d u\right)=:(r, z)
\end{aligned}
$$

transforms integral curves of the dynamical system to straight lines.
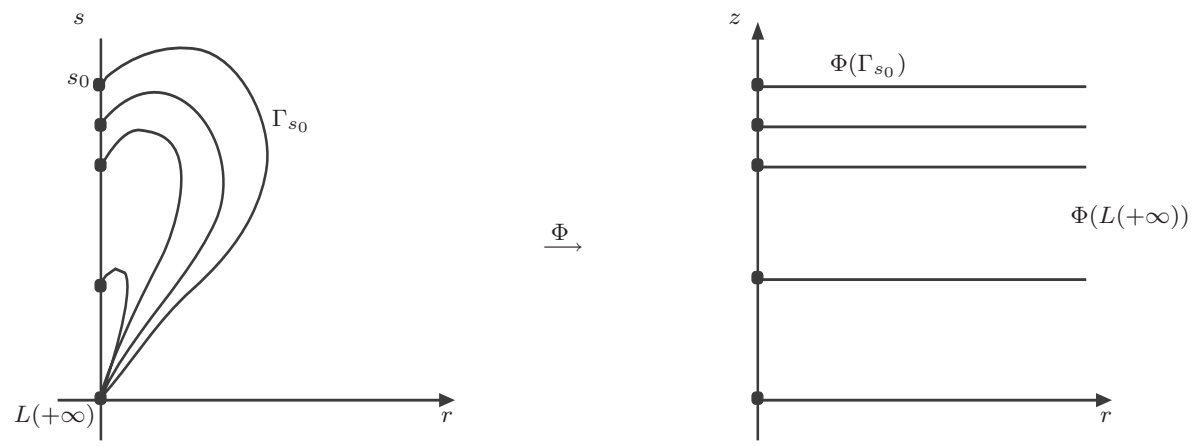

FiguRE 3. The coordinate transformation $\Phi$

\subsection{Description of the Poisson boundary}

The family $\left(\Gamma_{s_{0}}\right)_{s_{0} \in \mathbb{R}}$ when rotated about the symmetry axis (given by the vertical geodesic $L$ ) defines a foliation of $M$. If the idea is correct that Brownian motion exits $M$ asymptotically along the leaves of this foliation, then the $z$-component of Brownian motion (using the coordinates $(r, z, \alpha)$ as above) should have a large time limit. 
Theorem 4.5 (Arnaudon-Thalmaier-Ulsamer [5]). Considering Brownian motion on $M$ in the new coordinates:

$$
X=(R, S, A) \longmapsto X=(R, Z, A)
$$

where

$$
Z_{t}:=S_{t}-\int_{0}^{R_{t}} q(u) d u
$$

we find the following asymptotic behaviour: almost surely, as $t \rightarrow \zeta$,

$$
\left\{\begin{array}{l}
R_{t} \rightarrow \infty \\
Z_{t} \rightarrow Z_{\zeta} \in \mathbb{R} \\
A_{t} \rightarrow A_{\zeta} \in S^{1}
\end{array}\right.
$$

Moreover, the induced measures $\mathbb{P}_{x} \circ Z_{\zeta}^{-1}$ on $\mathbb{R}$, respectively, $\mathbb{P}_{x} \circ A_{\zeta}^{-1}$ on $S^{1}$ have full support.

Remark 4.6. For each $f \in C_{\mathrm{b}}\left(\mathbb{R} \times S^{1}\right), f \neq$ const,

$$
u(x)=\mathbb{E}_{x}\left[f\left(Z_{\zeta}, A_{\zeta}\right)\right]
$$

defines a non-trivial bounded harmonic function on $M$. Indeed, it can be shown that

$$
\mathscr{F}_{\text {inv }}=\sigma\left(Z_{\zeta}, A_{\zeta}\right)
$$

The proof of (4.2) is far from being obvious and uses time reversal arguments [28], coupling arguments for the time-reversed process, as well as a 0/1 law for the time-reversed process, see [5] for details.

Theorem 4.7 (Arnaudon-Thalmaier-Ulsamer [5]). Let $\mathscr{B}\left(\mathbb{R} \times S^{1} ; \mathbb{R}\right)$ be the set of bounded measurable functions on $\mathbb{R} \times S^{1}$, with the equivalence relation $f_{1} \simeq f_{2}$ if $f_{1}=f_{2}$ Lebesgue-a.e.

1. The map

$$
\begin{aligned}
\left(\mathscr{B}\left(\mathbb{R} \times S^{1} ; \mathbb{R}\right) / \simeq\right) & \longrightarrow \mathscr{H}_{b}(M) \\
f & \longmapsto\left(x \mapsto \mathbb{E}\left[f\left(Z_{\zeta}(x), A_{\zeta}(x)\right)\right]\right)
\end{aligned}
$$

is one to one.

2. The inverse map is given as follows. For $x \in M$, letting $K(x, \cdot, \cdot)$ be the density of $\left(Z_{\zeta}(x), A_{\zeta}(x)\right)$ with respect to the Lebesgue measure on $\mathbb{R} \times S^{1}$, for any $h \in \mathscr{H}_{b}(M)$ there exists a unique $f \in \mathscr{B}\left(\mathbb{R} \times S^{1} ; \mathbb{R}\right) / \simeq$ such that

$$
\forall x \in M, \quad h(x)=\int_{\mathbb{R} \times S_{1}} K(x, z, a) f(z, a) d z d a .
$$

Moreover, for $x \in M$, the kernel $K(x, \cdot, \cdot)$ in (4.3) is strictly positive a.e.

Note that the space of bounded harmonic functions $\mathscr{H}_{\mathrm{b}}(M)$ on the constructed manifold $M$ is as rich as in the pinched curvature case

$$
-a^{2} \leq \operatorname{Sect}^{M} \leq-b^{2}<0
$$


where one has

$$
\mathscr{F}_{\text {inv }}=\sigma\left(\Theta_{\infty}\right)
$$

The rich space of harmonic functions on $M$ comes however from completely different reasons. There is no contribution from the limiting angle of Brownian motion, since Brownian motion degenerates to a single point at infinity. To see the true Poisson boundary, the point $\infty_{M}$ at infinity has to be blown up to a twodimensional space.

Remark 4.8. From the point of view of Harnack inequalities harmonic functions in the constructed manifold share an interesting feature: on any neighbourhood in $M$ of the point $\infty_{M} \in S_{\infty}(M)$, a bounded harmonic function on $M$ attains each value between its global minimum and global maximum.

Remark 4.9. The constructed manifold has another interesting property in contrast to the pinched curvature case: it is easy to show that the $\sigma$-field of terminal events is strictly larger than the $\sigma$-field of shift-invariant events. This implies that the space of bounded harmonic functions is a proper subspace of the bounded space-time harmonic functions.

\subsection{Absolute continuity of the harmonic measure class}

It is interesting to note that on the constructed manifold, despite of diverging curvature, the harmonic measure has a density (Poisson kernel) with respect to the Lebesgue measure on the Poisson boundary $\mathbb{R} \times S^{1}$. In the pinched curvature case the harmonic measure may well be singular with respect to the surface measure on the sphere at infinity (see [21], [25], [19], [10] for results in this direction). Typically it is the fluctuation of the geometry at infinity which prevents harmonic measure from being absolutely continuous. It is well known that pinched curvature alone does in general not allow to bound the angular derivative of the Riemannian metric, when written in polar coordinates.

\subsection{Outlook}

The example discussed in this section can be modified in such a way that, with probability 1 , every point on $S_{\infty}(M)$ is a cluster point of $X_{t}$ (when $t \rightarrow \infty$ ). This can be achieved by properly moving the attracting point $\infty_{M}$ on the sphere at infinity. Such examples may turn out to be possible candidates for disproving Greene-Wu's conjecture.

\section{References}

[1] A. Ancona, Negatively curved manifolds, elliptic operators, and the Martin boundary, Ann. of Math. (2) 125 (1987), no. 3, 495-536.

[2] _ Convexity at infinity and Brownian motion on manifolds with unbounded negative curvature, Rev. Mat. Iberoamericana 10 (1994), no. 1, 189-220.

[3] M.T. Anderson, The Dirichlet problem at infinity for manifolds of negative curvature, J. Differential Geom. 18 (1983), no. 4, 701-721 (1984). 
[4] M.T. Anderson, R. Schoen, Positive harmonic functions on complete manifolds of negative curvature, Ann. of Math. (2) 121 (1985), no. 3, 429-461.

[5] M. Arnaudon, A. Thalmaier, S. Ulsamer, Existence of non-trivial harmonic functions on Cartan-Hadamard manifolds of unbounded curvature, Math. Z. 263 (2009), 369409.

[6] R.L. Bishop, B. O’Neill, Manifolds of Negative Curvature, Trans. Amer. Math. Soc. 145 (1968), 1-49.

[7] A. Borbély, The nonsolvability of the Dirichlet problem on negatively curved manifolds, Differential Geom. Appl. 8 (1998), no. 3, 217-237.

[8] H.I. Choi, Asymptotic Dirichlet problems for harmonic functions on Riemannian manifolds, Trans. Amer. Math. Soc. 281 (1984), no. 2, 691-716.

[9] M. Cranston, On specifying invariant $\sigma$-fields. Seminar on Stochastic Processes 1991, Birkhäuser, Progr. Probab., vol. 29 (1992), 15-37.

[10] M. Cranston, C. Mueller, A review of recent and older results on the absolute continuity of harmonic measure, Geometry of random motion (Ithaca, N.Y., 1987), Contemp. Math., vol. 73, Amer. Math. Soc., Providence, RI, 1988, pp. 9-19.

[11] P. Eberlein, B. O'Neill, Visibility Manifolds. Pacific J. Math. 46 (1973), no. 1, 45109.

[12] M. Émery, Stochastic calculus in manifolds, Universitext, Springer-Verlag, Berlin, 1989, With an appendix by P.-A. Meyer.

[13] R.E. Greene, H. Wu, Function theory on manifolds which possess a pole, Lecture Notes in Mathematics, vol. 699, Springer, Berlin, 1979.

[14] W. Hackenbroch, A. Thalmaier, Stochastische Analysis. Eine Einführung in die Theorie der stetigen Semimartingale, B. G. Teubner, Stuttgart, 1994.

[15] I. Holopainen, A. Vähäkangas, Asymptotic Dirichlet problem on negatively curved spaces. International Conference on Geometric Function Theory, Special Functions and Applications (R.W. Barnard and S. Ponnusamy, eds.), J. Analysis 15 (2007), 63-110.

[16] E.P. Hsu, Brownian motion and Dirichlet problems at infinity, Ann. Probab. 31 (2003), no. 3, 1305-1319.

[17] P. Hsu, W.S. Kendall, Limiting angle of Brownian motion in certain two-dimensional Cartan-Hadamard manifolds, Ann. Fac. Sci. Toulouse Math. (6) 1 (1992), no. 2, 169186.

[18] P. Hsu, P. March, The limiting angle of certain Riemannian Brownian motions, Comm. Pure Appl. Math. 38 (1985), no. 6, 755-768.

[19] A. Katok, Four applications of conformal equivalence to geometry and dynamics, Ergodic Theory Dynam. Systems 8* (1988), no. Charles Conley Memorial Issue, 139-152.

[20] W.S. Kendall, Brownian motion on a surface of negative curvature, Seminar on probability, XVIII, Lecture Notes in Math., vol. 1059, Springer, Berlin, 1984, pp. $70-$ 76.

[21] Ju.I. Kifer, Brownian motion and harmonic functions on manifolds of negative curvature, Theor. Probability Appl. 21 (1976), no. 1, 81-95. 
[22] Brownian motion and positive harmonic functions on complete manifolds of nonpositive curvature, From local times to global geometry, control and physics (Coventry, 1984/85), Pitman Res. Notes Math. Ser., vol. 150, Longman Sci. Tech., Harlow, 1986, pp. 187-232.

[23] H. Le, Limiting angle of Brownian motion on certain manifolds, Probab. Theory Related Fields 106 (1996), no. 1, 137-149.

$[24] \_$, Limiting angles of $\Gamma$-martingales, Probab. Theory Related Fields 114 (1999), no. 1, 85-96.

[25] F. Ledrappier, Propriété de Poisson et courbure négative, C. R. Acad. Sci. Paris Sér. I Math. 305 (1987), no. 5, 191-194.

[26] P. March, Brownian motion and harmonic functions on rotationally symmetric manifolds, Ann. Probab. 11 (1986) 793-801.

[27] F. Mouton, Comportement asymptotique des fonctions harmoniques en courbure négative, Comment. Math. Helv. 70 (1995), 475-505.

[28] É. Pardoux, Grossissement d'une filtration et retournement du temps d'une diffusion, Séminaire de Probabilités, XX, 1984/85, Lecture Notes in Math., vol. 1204, Springer, Berlin, 1986, pp. 48-55.

[29] J.-J. Prat, Étude asymptotique du mouvement brownien sur une variété riemannienne à courbure négative, C. R. Acad. Sci. Paris Sér. A-B 272 (1971), A1586-A1589.

[30] _ Étude asymptotique et convergence angulaire du mouvement brownien sur une variété à courbure négative, C. R. Acad. Sci. Paris Sér. A-B 280 (1975), A1539A1542.

[31] D. Sullivan, The Dirichlet problem at infinity for a negatively curved manifold, J. Differential Geom. 18 (1983), no. 4, 723-732 (1984).

[32] A. Vähäkangas, Dirichlet problem at infinity for $\mathcal{A}$-harmonic functions, Potential Anal. 27 (2007), no. 1, 27-44.

[33] H.H. Wu, Function theory on noncompact Kähler manifolds, Complex differential geometry, DMV Sem., vol. 3, Birkhäuser, Basel, 1983, pp. 67-155.

Marc Arnaudon

Laboratoire de Mathématiques et Applications, CNRS: UMR6086

Université de Poitiers, Téléport 2 - BP 30179

F-86962 Futuroscope Chasseneuil Cedex, France

e-mail: arnaudon@math.univ-poitiers.fr

Anton Thalmaier

Unité de Recherche en Mathématiques, FSTC

Université du Luxembourg

6, rue Richard Coudenhove-Kalergi

L-1359 Luxembourg, Grand-Duchy of Luxembourg

e-mail: anton.thalmaier@uni.lu 